[Chem. Pharm. Bull.

36(11)4453-4461(1988)]

\title{
Limonoids and Quinolone Alkaloids from Evodia rutaecarpa BENTHAM
}

\author{
Tohru Sugimoto, Toshio Miyase, Masanori Kuroyanagi, \\ and AKIRA Ueno*
}

School of Pharmaceutical Sciences, University of Shizuoka,

2-2-1, Oshika, Shizuoka 422, Japan

(Received May 7, 1988)

\begin{abstract}
Four new limonoids and five new quinolone alkaloids were isolated from the fruit of Evodia rutaecarpa BENTHAM (Rutaceae), together with seven known limonoids and four known quinolone alkaloids, and their structures were determined on the basis of spectral data and chemical reactions.

Keywords-limonoid; quinolone alkaloid; Evodia rutaecarpa; $12 \alpha$-hydroxylimonin; $12 \alpha$ hydroxyevodol; 1-methyl-2-[(Z)-6-undecenyl]-4(1H)-quinolone; 1-methyl-2-[(Z)-10-pentadecenyl]$4(1 H)$-quinolone; 1-methyl-2-[( $Z$ )-6-pentadecenyl]-4(1 H )-quinolone; 1-methyl-2-[(6Z,9Z)6,9-pentadecadienyl]-4(1 H)-quinolone; 1-methyl-2-[(4Z,7Z)-4,7-tridecadienyl]-4(1 H )-quinolone
\end{abstract}

In the course of our studies on the biologically active compounds of Rutaceous plants, we have investigated in particular the limonoids and quinolone alkaloids in Evodia rutaecarpa BENTHAM. Limonoids were detected as brown-orange spots on thin layer chromatography (TLC) with Ehrlich's reagent, which reacts with the furan ring. ${ }^{1)}$ From the fruit of this plant, seven known limonoids, limonin (I), ${ }^{2)}$ rutaevine (II), ${ }^{3)}$ evodol (III), ${ }^{4)}$ obacunone (IV), ${ }^{5)}$ jangomolide $(\mathrm{V}),{ }^{6)}$ rutaevine acetate $(\mathrm{VI})^{7)}$ and graucin $\mathrm{A}(\mathrm{VII}),{ }^{8)}$ were isolated together with new limonoids, 12 $\alpha$-hydroxylimonin (VIII), 12 $\alpha$-hydroxyevodol (IX), $6 \alpha$-acetoxy-5epilimonin (X) and 6 $\beta$-acetoxy-5-epilimonin (XI). Compounds X and XI were reported in our
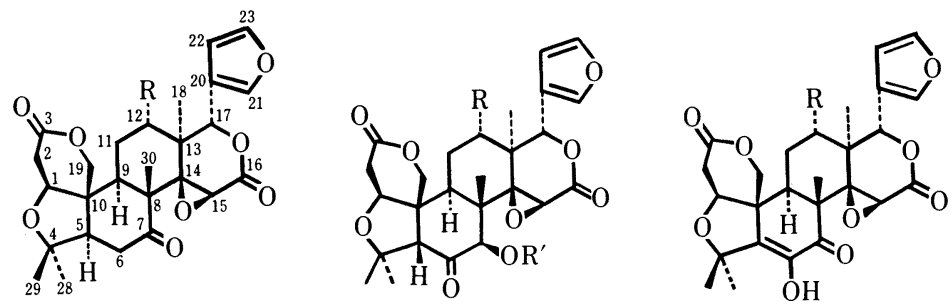

$$
\mathrm{I}: \mathrm{R}=\mathrm{H}
$$

VIII $: \mathrm{R}=\mathrm{OH}$

II : $\mathrm{R}=\mathrm{H}, \mathrm{R}^{\prime}=\mathrm{H}$

VI $: \mathrm{R}=\mathrm{H}, \mathrm{R}^{\prime}=\mathrm{Ac}$

VII : $\mathrm{R}=\mathrm{OH}, \mathrm{R}^{\prime}=\mathrm{H}$

III $: \mathrm{R}=\mathrm{H}$

IX : $\mathrm{R}=\mathrm{OH}$

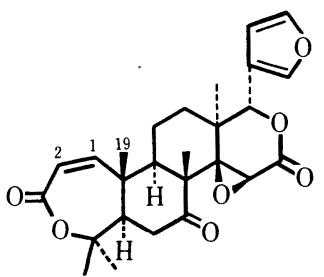

IV

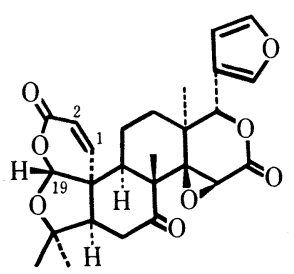

V

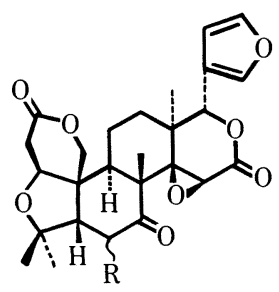

$\mathrm{X}: \mathrm{R}=\alpha-\mathrm{OAc}$ $\mathrm{XI}: \mathrm{R}=\beta-\mathrm{OAc}$

Chart 1 
previous paper. ${ }^{9}$ This paper describes two new limonoids, VIII and IX, and quinolone alkaloids.

$12 \alpha$-Hydroxylimonin (VIII) was obtained as a colorless powder, $[\alpha]_{\mathrm{D}}^{20}-134.0^{\circ}(c=1.00$, $\left.\mathrm{CHCl}_{3}\right)$. The infrared (IR) spectrum showed the presence of hydroxyl $\left(3475 \mathrm{~cm}^{-1}\right)$, lactone $\left(1750 \mathrm{~cm}^{-1}\right)$ and ketone $\left(1720 \mathrm{~cm}^{-1}\right)$ groups. The electron impact mass spectrum (EI-MS) showed fragment ion peaks at $m / z 485(\mathrm{M}-\mathrm{H})^{+}, 471\left(\mathrm{M}-\mathrm{CH}_{3}\right)^{+}$. The proton nuclear magnetic resonance $\left({ }^{1} \mathrm{H}-\mathrm{NMR}\right)$ spectrum exhibited signals due to four tertiary methyl groups at $\delta 0.96,1.13,1.16$ and 1.28 , two singlet signals at $\delta 3.80(15-\mathrm{H})$ and $5.44(17-\mathrm{H})$, an $\mathrm{AX}$ quartet $(J=13 \mathrm{~Hz})$ at $\delta 4.40\left(19 \mathrm{H}_{\alpha}\right)$ and $4.82\left(19-\mathrm{H}_{\beta}\right)$, a $\beta$-substituted furan at $\delta 6.46(22-\mathrm{H})$ and $7.50(2 \mathrm{H}, 21-, 23-\mathrm{H})$, and a carbinol methine signal at $\delta 3.94(\mathrm{~d}, J=5 \mathrm{~Hz}, 12-\mathrm{H})$. These ${ }^{1} \mathrm{H}-\mathrm{NMR}$ spectral features closely resembled those of limonin (I) except for the presence of the signals due to an additional hydroxyl group. In the carbon-13 nuclear magnetic resonance $\left({ }^{13} \mathrm{C}-\mathrm{NMR}\right)$ spectrum, the signals of the C-ring of VIII were similar to those of VII compared with rutaevine (II) ${ }^{8}$ (see Fig. 1). Compound VIII was substituted with a hydroxyl group at the 12-position of limonin. In order to decide the stereostructure at 12-C, VIII was acetylated. In the ${ }^{1} \mathrm{H}$-NMR spectrum, the acetyl methyl signal of VIII acetate was observed at $\delta 1.76$, because the acetyl methyl was shielded heavily by paramagnetic anisotropy of the furan ring ${ }^{8}$ (see Fig. 2). Thus, the hydroxyl group of at 12-C was $\alpha$.

$12 \alpha$-Hydroxyevodol (IX) was obtained as an amorphous solid, $[\alpha]_{\mathrm{D}}^{20}-114.0^{\circ}(c=0.48$, $\mathrm{MeOH})$. The IR spectrum showed the presence of hydroxyl $\left(3450 \mathrm{~cm}^{-1}\right)$, lactone $\left(1750 \mathrm{~cm}^{-1}\right)$ and conjugated ketone $\left(1690 \mathrm{~cm}^{-1}\right)$ groups. The EI-MS showed the $\mathrm{M}^{+}$peaks at $\mathrm{m} / z 500$ and a fragment ion peak at $m / z 485\left(\mathrm{M}-\mathrm{CH}_{3}\right)^{+}$. The ${ }^{1} \mathrm{H}-\mathrm{NMR}$ spectrum exhibited signals due to four tertiary methyl groups at $\delta 1.02,1.10,1.51$ and 1.57 , three singlet signals at $\delta 4.10(15-\mathrm{H})$, $5.46(17-\mathrm{H})$ and $4.67\left(2 \mathrm{H}, 19-\mathrm{H}_{2}\right)$, a $\beta$-substituted furan at $\delta 6.46(22-\mathrm{H})$ and $7.54(2 \mathrm{H}, 21-, 23-$ $\mathrm{H})$, and a carbinol methine signal at $\delta 3.94(\mathrm{~d}, J=5 \mathrm{~Hz}, 12-\mathrm{H})$. These ${ }^{1} \mathrm{H}-\mathrm{NMR}$ spectral features closely resembled those of evodol (III) except for the presence of the signals due to an additional hydroxyl group (see Fig. 1). The ${ }^{13} \mathrm{C}$-NMR spectrum showed the hydroxyl group attached at the 12-position of III, as in VIII. The $\alpha$ configuration was suggested, because the acetyl methyl signal at 12-C of IX acetate was observed at $\delta 1.74$.
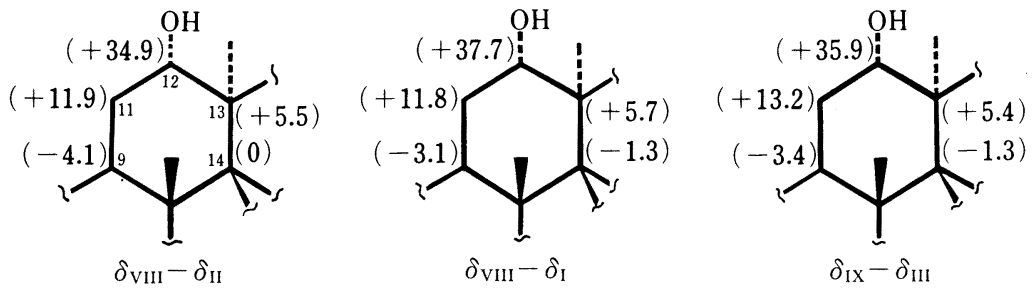

Fig. 1. Difference of ${ }^{13} \mathrm{C}$-NMR Chemical Shifts on the C-Ring between 12 Hydroxy Derivatives and Base Compounds

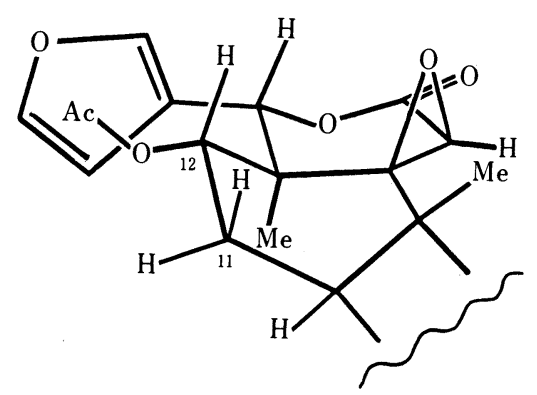

Fig. 2. Partial Structure of $12 \alpha$-Acetoxyl Limonoids 
HPLC analysis
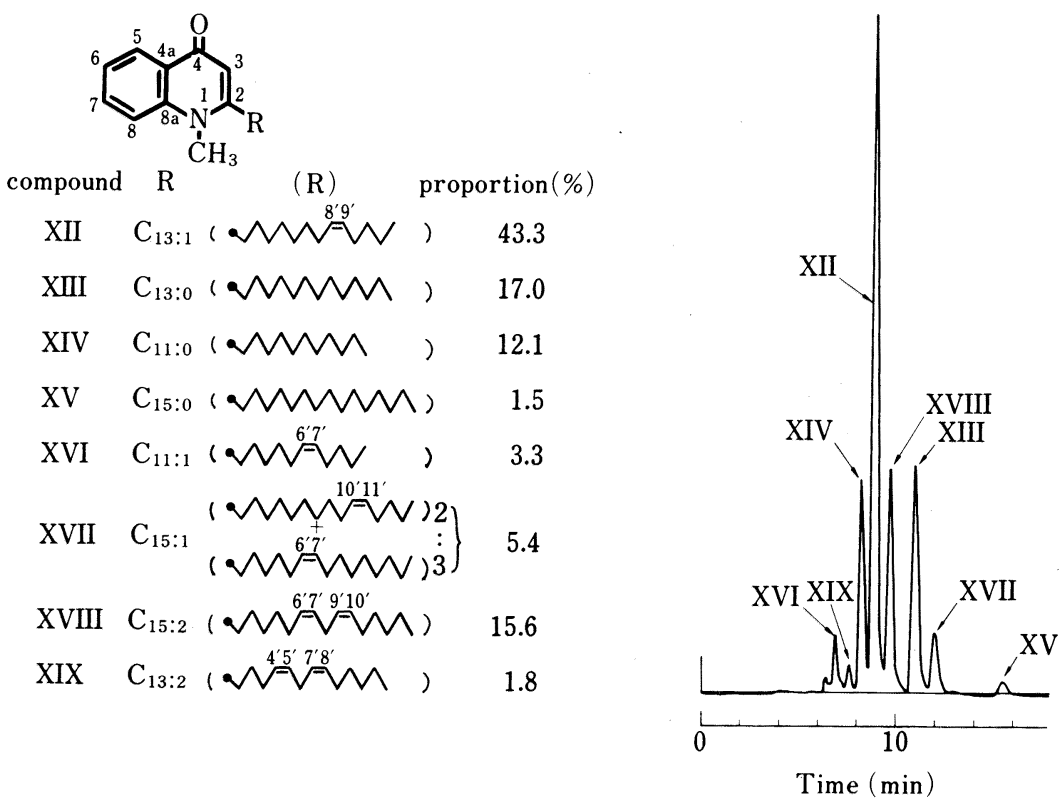

Fig. 3. Quinolone Alkaloid Fraction Analysis

HPLC analysis: column Develosil C8-7; solvent $\mathrm{MeOH}-\mathrm{H}_{2} \mathrm{O}$ (90: 10); wavelength $321 \mathrm{~nm}$; flow rate $1.0 \mathrm{ml} / \mathrm{min}$.
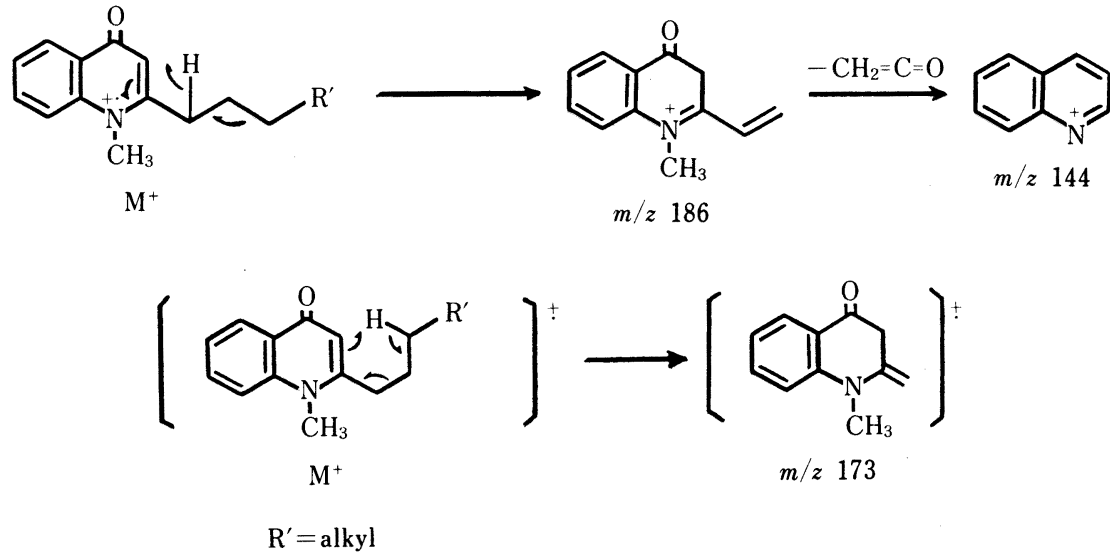

Fig. 4. Characteristic Mass Spectral Fragment Ions of Quinolone Alkaloids

Quinolone alkaloids were detected as purple spots with hydrogen hexachloroplatinate reagent on TLC. Analysis of the quinolone alkaloid fraction on high performance liquid chromatography (HPLC) showed eight peaks (Fig. 3). Four of them were known compounds; they were identified as evocarpine (XII), ${ }^{10)}$ dihydroevocarpine (XIII), 1-methyl-2-undecyl$4(1 H)$-quinolone $(\mathrm{XIV})$ and 1-methyl-2-pentadecyl-4( $1 H)$-quinolone $(\mathrm{XV}){ }^{1{ }^{11}}$ The remaining four were identified as follows.

1-Methyl-2-[(Z)-6-undecyl]-4(1H)-quinolone (XVI) was obtained as a colorless oil. The ultraviolet (UV) spectrum showed the same absorptions as evocarpine (XII). ${ }^{10)}$ The EI-MS showed the $\mathrm{M}^{+}$peak at $\mathrm{m} / z 311$ and the fragment ion peaks arising from McLafferty 
rearrangement of the molecular ion at $m / z 173$, from displacement rearrangement of the molecular ion at $m / z 186$, and elimination of the ketene at $m / z 144^{10)}$ (see Fig. 4). The ${ }^{1} \mathrm{H}$ NMR spectrum exhibited signals due to the quinolone skeleton, i.e., $\mathrm{N}$-methyl group at $\delta$ 3.73 , conjugated olefinic proton at $\delta 6.22(1 \mathrm{H}, \mathrm{s}, 3-\mathrm{H})$, aromatic protons at $\delta 7.3-7.8(3 \mathrm{H}, \mathrm{m}$, $6-, 7-, 8-\mathrm{H})$, aromatic peri-proton at $\delta 8.41(1 \mathrm{H}, \mathrm{dd}, J=8,2 \mathrm{~Hz}, 5-\mathrm{H})$, and olefinic protons at $\delta$ $5.35\left(2 \mathrm{H}, \mathrm{m}, 6^{\prime}-, 7^{\prime}-\mathrm{H}\right)$. On catalytic hydrogenation, XVI was converted to XIV. This suggested that the side chain carbon number was eleven. On Lemieux-Johnson oxidation, XVI produced pentanal which was identified by comparing its 2,4-dinitrophenylhydrazone with an authentic sample. This suggested that the double bond of the side chain existed at the $6^{\prime}$-position, and it was considered to be in $Z$-form based on the ${ }^{13} \mathrm{C}$-NMR chemical shift of the allyl carbon $(\delta$ 27.0).

Compound XVII was obtained as a colorless oil. The UV and ${ }^{1} \mathrm{H}-\mathrm{NMR}$ spectra exhibited signals due to the quinolone skeleton. The EI-MS showed the $\mathbf{M}^{+}$peak at $\mathrm{m} / z 367$ and fragment ion peaks at $m / z 186,173,144$. The ${ }^{1} \mathrm{H}-\mathrm{NMR}$ spectrum also exhibited a signal due to olefinic protons at $\delta 5.40(2 \mathrm{H}$, br t, $J=5 \mathrm{~Hz})$. On catalytic hydrogenation, XVII was converted to $\mathrm{XV}$. This suggested that the side chain carbon number was fifteen. However, the ${ }^{13} \mathrm{C}-\mathrm{NMR}$ spectrum indicated that XVII consisted of two compounds. This was proved by

TABLE I. ${ }^{1} \mathrm{H}-\mathrm{NMR}$ Chemical Shifts of Limonoids

\begin{tabular}{|c|c|c|c|c|c|c|c|c|c|}
\hline & $\mathrm{I}^{a, i)}$ & $\mathrm{II}^{b, j)}$ & $\mathrm{III}^{a, i)}$ & $\mathrm{IV}^{a, i)}$ & $\mathrm{V}^{a, i}$ & $\mathrm{VI}^{a, j)}$ & $\mathrm{VII}^{b, j)}$ & $\mathrm{VIII}^{a, j)}$ & $\mathrm{IX}^{a, j)}$ \\
\hline 1 & $\begin{array}{l}4.04 \\
\text { (brs) }\end{array}$ & $\begin{array}{l}4.54 \\
\text { (br s) }\end{array}$ & $\begin{array}{l}4.07 \\
\text { (brs) }\end{array}$ & $\begin{array}{l}6.51 \\
(d, 11)\end{array}$ & $\begin{array}{l}6.52 \\
(d, 10)\end{array}$ & $\begin{array}{l}4.40 \\
\text { (brs) }\end{array}$ & $\begin{array}{l}4.37 \\
\text { (brs) }\end{array}$ & $\begin{array}{l}4.80 \\
\text { (brs) }\end{array}$ & $\begin{array}{l}4.12 \\
\text { (brs) }\end{array}$ \\
\hline $2 \alpha$ & $\begin{array}{l}2.68 \\
(\mathrm{dd}, 17,2)\end{array}$ & $\begin{array}{l}2.64 \\
(\mathrm{dd}, 15,3)\end{array}$ & $\begin{array}{l}2.84 \\
(\mathrm{dd}, 17,5)\end{array}$ & 5.97 & 6.13 & $\begin{array}{l}2.64 \\
(d d, 16,3)\end{array}$ & ${ }^{k)}$ & ${ }^{k)}$ & $-{ }^{k)}$ \\
\hline $2 \beta$ & $\begin{array}{l}2.98 \\
(\mathrm{dd}, 17,4)\end{array}$ & $\begin{array}{l}2.92 \\
(\mathrm{dd}, 15,3)\end{array}$ & $\begin{array}{l}2.97 \\
(\mathrm{dd}, 17,5)\end{array}$ & $(d, 11)$ & $(\mathrm{d}, 10)$ & $\begin{array}{l}2.90 \\
(\mathrm{dd}, 16,2.5)\end{array}$ & $-{ }^{k)}$ & $-{ }^{k)}$ & $-^{k)}$ \\
\hline 5 & $\begin{array}{l}2.23 \\
(\mathrm{dd}, 15,3)\end{array}$ & $2.86(\mathrm{~s})$ & & $\begin{array}{l}2.61 \\
(\mathrm{dd}, 11,4)\end{array}$ & $\begin{array}{l}2.68 \\
(t, 6)\end{array}$ & $3.06(\mathrm{~s})$ & $2.95(\mathrm{~s})$ & $-^{k)}$ & \\
\hline $6 \alpha$ & $\begin{array}{l}2.47 \\
(\mathrm{dd}, 14,3)\end{array}$ & & & $\begin{array}{l}2.30 \\
(\mathrm{dd}, 11,4)\end{array}$ & 2.70 & & & $-^{k)}$ & \\
\hline $6 \beta$ & $\begin{array}{l}2.86 \\
(\mathrm{dd}, 15,14)\end{array}$ & & & $\begin{array}{l}2.99 \\
(t, 11)\end{array}$ & $(d, 6)$ & & & & \\
\hline 7 & & $\begin{array}{l}4.25 \\
(d, 4.5)\end{array}$ & & & & $5.63(\mathrm{~s})$ & $\begin{array}{l}5.81 \\
(\mathrm{~d}, 5)\end{array}$ & & \\
\hline 9 & $\begin{array}{l}2.55 \\
(\mathrm{dd}, 12,3)\end{array}$ & $-{ }^{k)}$ & $\begin{array}{l}2.64 \\
(\mathrm{dd}, 13,3)\end{array}$ & $\begin{array}{l}2.15 \\
(\mathrm{dd}, 8.5,3.5)\end{array}$ & $\begin{array}{l}2.66 \\
(t, 8)\end{array}$ & $-^{k)}$ & ${ }^{k)}$ & $-^{k)}$ & $-^{k)}$ \\
\hline $12 \beta$ & ${ }^{k)}$ & $-^{k)}$ & ${ }^{k)}$ & $-^{k)}$ & $\ldots{ }^{k}$ & $-{ }^{k)}$ & $\begin{array}{l}4.93 \\
(d, 4)\end{array}$ & $\begin{array}{l}3.94 \\
(d, 5)\end{array}$ & $\begin{array}{l}3.94 \\
(d, 5)\end{array}$ \\
\hline 15 & $4.04(\mathrm{~s})$ & $4.00(\mathrm{~s})$ & 4.12 (s) & $3.66(\mathrm{~s})$ & $3.99(\mathrm{~s})$ & $3.84(\mathrm{~s})$ & $4.01(\mathrm{~s})$ & $3.80(\mathrm{~s})$ & $4.10(\mathrm{~s})$ \\
\hline 17 & $5.48(\mathrm{~s})$ & $5.48(\mathrm{~s})$ & $5.43(\mathrm{~s})$ & $5.46(\mathrm{~s})$ & & $5.50(\mathrm{~s})$ & $5.59(\mathrm{~s})$ & $5.44(\mathrm{~s})$ & $5.46(\mathrm{~s})$ \\
\hline $19 \alpha$ & 4.46 & & 4.62 & & $5.53(\mathrm{~s})$ & 4.16 & & 4.40 & \\
\hline $19 \beta$ & $\begin{array}{l}(\mathrm{d}, 13) \\
4.77 \\
(\mathrm{~d}, 13)\end{array}$ & 4.3 (br s) & $\begin{array}{l}(d, 13) \\
4.66 \\
(d, 13)\end{array}$ & $1.24(\mathrm{~s})$ & $6.07(\mathrm{~s})$ & $\begin{array}{l}(\mathrm{d}, 13) \\
4.34 \\
(\mathrm{~d}, 13)\end{array}$ & 3.94 .3 & $\begin{array}{l}(\mathrm{d}, 13) \\
4.82 \\
(\mathrm{~d}, 13)\end{array}$ & $4.67(\mathrm{~s})$ \\
\hline 21 & 7.41 & 7.70 & $7.41^{c)}$ & 7.43 & 7.40 & 7.44 & $7.84^{e)}$ & 7.50 & 7.54 \\
\hline 22 & 6.38 & 6.55 & 6.38 & 6.37 & 6.32 & 6.38 & 6.61 & 6.46 & 6.46 \\
\hline 23 & 7.40 & 7.77 & $7.40^{c)}$ & 7.40 & 7.41 & 7.44 & $7.73^{e)}$ & 7.50 & 7.54 \\
\hline 18 & $1.17(\mathrm{~s})$ & $1.35(\mathrm{~s})$ & 1.04 (s) & $1.12(\mathrm{~s})$ & $1.16(\mathrm{~s})$ & $1.32(\mathrm{~s})^{d)}$ & $1.18(\mathrm{~s})^{f)}$ & $1.16(\mathrm{~s})^{g)}$ & $1.51^{h)}$ \\
\hline 28 & $1.30(\mathrm{~s})$ & $1.13(\mathrm{~s})$ & $1.54(\mathrm{~s})$ & $1.46(\mathrm{~s})$ & $1.36(\mathrm{~s})$ & $1.32(\mathrm{~s})^{d)}$ & $1.13(\mathrm{~s})^{f)}$ & $1.13(\mathrm{~s})^{g)}$ & $1.57^{h)}$ \\
\hline 29 & $1.18(\mathrm{~s})$ & $1.25(\mathrm{~s})$ & 1.49 (s) & $1.51(\mathrm{~s})$ & $1.30(\mathrm{~s})$ & $1.40(\mathrm{~s})^{d)}$ & $1.28(\mathrm{~s})^{f)}$ & $1.28(\mathrm{~s})^{g)}$ & $1.10^{h)}$ \\
\hline 30 & 1.07 (s) & $0.57(\mathrm{~s})$ & $1.16(\mathrm{~s})$ & $1.51(\mathrm{~s})$ & $1.30(\mathrm{~s})$ & $0.80(\mathrm{~s})^{d)}$ & $0.56(\mathrm{~s})^{f)}$ & $0.96(\mathrm{~s})^{g)}$ & $1.02^{h)}$ \\
\hline
\end{tabular}

a) In $\mathrm{CDCl}_{3} . \quad$ b) In DMSO- $d_{6} . \quad c-h$ ) Assignments may be interchanged in each column. i) Measured at $400 \mathrm{MHz}$. j) Measured at $90 \mathrm{MHz} . k$ ) Could not be assigned. 
TABLE II. ${ }^{13}$ C-NMR Chemical Shifts of Limonoids

\begin{tabular}{|c|c|c|c|c|c|c|c|c|c|}
\hline & $\mathrm{I}^{a)}$ & $\mathrm{II}^{b)}$ & $\mathrm{III}^{b)}$ & $\mathrm{IV}^{a)}$ & $\mathrm{V}^{a)}$ & $\mathrm{VI}^{b)}$ & $\mathrm{VII}^{b)}$ & VIII $^{a)}$ & $\mathrm{IX}^{b)}$ \\
\hline 1 & 79.1 & 81.9 & 78.7 & 156.8 & 150.7 & 81.8 & 81.8 & 78.9 & 79.0 \\
\hline 2 & 35.7 & 35.9 & 35.0 & 123.0 & 119.0 & 35.8 & 35.9 & $35.7^{h)}$ & 35.0 \\
\hline 3 & 169.1 & 170.9 & 169.8 & $166.9^{e)}$ & 160.3 & 170.6 & 170.7 & 169.7 & 169.7 \\
\hline 4 & 80.3 & 81.4 & 81.8 & 84.0 & 86.3 & 81.5 & 81.5 & 80.2 & 81.1 \\
\hline 5 & 60.5 & 64.5 & 140.7 & 57.4 & 53.3 & 64.4 & 64.7 & 61.1 & 140.3 \\
\hline 6 & 36.4 & 207.2 & 142.3 & 40.0 & 37.7 & 200.4 & 207.2 & $36.5^{h)}$ & 141.9 \\
\hline 7 & 206.2 & 81.4 & 195.3 & 207.5 & 209.6 & 81.5 & 81.8 & 206.0 & 195.3 \\
\hline 8 & 51.3 & $48.6^{c)}$ & $47.1^{d)}$ & 53.0 & 49.3 & $48.3^{f)}$ & $48.1^{g)}$ & 52.3 & 47.3 \\
\hline 9 & 48.1 & $45.3^{c)}$ & 46.2 & 49.3 & 41.8 & $43.8^{f)}$ & 41.2 & 45.0 & 42.8 \\
\hline 10 & 45.9 & $45.6^{()}$ & $46.6^{d)}$ & 43.3 & 49.3 & $46.0^{f)}$ & $45.8^{g)}$ & 45.8 & 46.9 \\
\hline 11 & 18.9 & 19.3 & 18.7 & 17.1 & 19.2 & 19.1 & 31.2 & 30.7 & 31.9 \\
\hline 12 & 30.8 & 31.0 & 30.0 & 32.8 & 29.1 & 30.8 & 65.9 & 68.5 & 65.9 \\
\hline 13 & 37.9 & 37.2 & 36.8 & 37.5 & 38.5 & 37.2 & 42.9 & 43.6 & 42.2 \\
\hline 14 & 65.7 & 65.9 & 65.7 & 65.1 & 67.2 & 65.4 & 65.9 & 64.4 & 64.4 \\
\hline 15 & 53.9 & 50.8 & 51.9 & 53.4 & 54.2 & 50.2 & 50.6 & 52.4 & 50.6 \\
\hline 16 & 166.9 & 166.9 & 166.7 & $166.7^{e)}$ & 166.6 & 166.5 & 166.8 & 166.2 & 166.5 \\
\hline 17 & 77.8 & 77.5 & 77.2 & 78.0 & 77.9 & 77.5 & 77.0 & 76.8 & 76.6 \\
\hline 19 & 65.4 & 69.8 & 69.5 & - & 104.0 & 69.3 & 70.1 & 65.9 & 69.3 \\
\hline 20 & 120.0 & 120.0 & 119.8 & 120.0 & 120.0 & 119.7 & 119.8 & 120.0 & 119.7 \\
\hline 21 & 141.1 & 141.6 & 141.6 & 141.1 & 141.2 & 141.6 & 141.7 & 141.6 & 141.6 \\
\hline 22 & 109.7 & 110.3 & 110.0 & 109.8 & 109.7 & 110.1 & 110.6 & 109.6 & 110.4 \\
\hline 23 & 143.2 & 143.4 & 143.4 & 143.3 & 143.3 & 143.4 & 143.0 & 144.3 & 142.9 \\
\hline \multirow{5}{*}{ C-Methyls } & 17.6 & 14.7 & 17.5 & 16.5 & 15.7 & 14.6 & 14.1 & 13.4 & 12.9 \\
\hline & 20.7 & 20.5 & 19.8 & 19.6 & 20.2 & 20.3 & 14.7 & 16.7 & 17.5 \\
\hline & 21.4 & 23.8 & 24.9 & 21.2 & 24.9 & 23.3 & 23.8 & 21.5 & 25.0 \\
\hline & 30.2 & 28.8 & 25.5 & 26.9 & 31.9 & 28.5 & 28.7 & 30.1 & 25.5 \\
\hline & & & & 32.1 & & & & & \\
\hline Acetate carbonyl & & & & & & 169.5 & & & \\
\hline Acetate methyl & & & & & & 20.0 & & & \\
\hline
\end{tabular}

a) In $\left.\mathrm{CDCl}_{3} . \quad b\right)$ In DMSO- $\left.d_{6} . \quad c-h\right)$ Assignments may be interchanged in each column.

Lemieux-Johnson oxidation, in which XVII produced pentanal and nonanal in the proportion of $2: 3$. The double bond was elucidated as $Z$-form based on the ${ }^{13} \mathrm{C}-\mathrm{NMR}$ chemical shifts of allyl carbon $(\delta 26.9,27.0)$. Consequently, XVII was a mixture of 1-methyl-2-[(Z)-10pentadecenyl]-4(1H)-quinolone and 1-methyl-2-[( $Z$ )-6-pentadecenyl]-4(1H)-quinolone in the proportion mentioned above.

1-Methyl-2-[(6Z,9Z)-6,9-pentadecadienyl]-4(1H)-quinolone (XVIII) was obtained as a colorless oil. The UV and ${ }^{1} \mathrm{H}-\mathrm{NMR}$ spectra again exhibited signals due to the quinolone skeleton. The EI-MS showed the $\mathrm{M}^{+}$peak at $\mathrm{m} / \mathrm{z} 365$ and the fragment ion peaks at $\mathrm{m} / \mathrm{z} 186$, 173 and 144. In addition, the ${ }^{1} \mathrm{H}-\mathrm{NMR}$ spectrum exhibited the signals due to olefinic protons at $\delta 5.40\left(4 \mathrm{H}, \mathrm{m}, 6^{\prime}-, 7^{\prime}-, 9^{\prime}-, 1^{\prime}-\mathrm{H}\right)$. On catalytic hydrogenation, XVIII was converted to XV. This suggested that the side chain carbon number was fifteen. On Lemieux-Johnson oxidation, XVIII produced hexanal and malonaldehyde. This suggested that the double bonds of the side chain existed at the 6'- and 9'-positions, and were showed $Z$-form, based on the ${ }^{13} \mathrm{C}-\mathrm{NMR}$ spectrum chemical shifts of allyl carbon $(\delta 26.9,25.5$ and 27.0$)$, as in linoleic acid.

1-Methyl-2-[(4Z,7Z)-4,7-tridecadienyl]-4(1H)-quinolone (XIX) was obtained as a colorless oil. Each spectral data showed similarity between XIX and XVIII. On catalytic hydrogenation, XIX was converted to XIII. This suggested that the side chain carbon number was thirteen. On Lemieux-Johnson oxidation, XIX produced hexanal and malonaldehyde. 
TABLE III. ${ }^{13}$ C-NMR Chemical Shifts of Quinolone Alkaloids and Linoleic Acid in $\mathrm{CDCl}_{3}$

\begin{tabular}{|c|c|c|c|c|c|c|c|c|c|c|}
\hline & XII & XIII & XIV & $X V$ & XVI & XVII & XVIII & XIX & \multicolumn{2}{|c|}{ Linoleic acid } \\
\hline 2 & 154.5 & 154.7 & 154.7 & 155.2 & 154.6 & 154.7 & 154.5 & 154.5 & 1 & 180.2 \\
\hline 3 & 110.5 & 111.1 & 111.1 & 111.1 & 111.2 & 110.7 & 110.7 & 111.2 & 2 & 34.2 \\
\hline 4 & 177.3 & 177.7 & 177.7 & 178.0 & 177.8 & 177.4 & 177.4 & 177.9 & 3 & 24.8 \\
\hline $4 a$ & a) $^{a}$ & a) & $-^{a)}$ & ${ }^{a}$ & $\left.\ldots^{a}\right)$ & -a) & -a) & -a) $^{a}$ & 4 & $29.2^{r)}$ \\
\hline 5 & 126.0 & 126.6 & 126.6 & 126.8 & 126.7 & 126.2 & 126.2 & 126.8 & 5 & $29.2^{r 1}$ \\
\hline 6 & 122.8 & 123.2 & 123.2 & 123.5 & 123.3 & 123.0 & 122.9 & 123.4 & 6 & $29.2^{r)}$ \\
\hline 7 & 131.7 & 132.0 & 132.0 & 132.2 & 132.0 & 131.8 & 131.7 & 132.1 & 7 & $29.7^{r)}$ \\
\hline 8 & 115.2 & 115.3 & 115.3 & 115.4 & 115.3 & 115.3 & 115.2 & 115.3 & 8 & 27.3 \\
\hline \multirow[t]{2}{*}{$8 \mathrm{a}$} & 141.6 & 142.0 & 142.0 & 142.0 & 142.0 & 141.7 & 141.7 & - $^{a)}$ & 9 & $130.0^{s)}$ \\
\hline & & & & & & & & & 10 & $127.4^{s)}$ \\
\hline \multirow[t]{2}{*}{$\mathrm{N}-\mathrm{CH}_{3}$} & 33.8 & 34.1 & 34.1 & 34.3 & 34.1 & 34.0 & 33.9 & 34.2 & 11 & 25.8 \\
\hline & & & & & & & & & 12 & $128.2^{s)}$ \\
\hline $1^{\prime}$ & 34.3 & 34.7 & 34.7 & 34.9 & 34.7 & 34.5 & 34.5 & 34.2 & 13 & $130.3^{s)}$ \\
\hline $2^{\prime}$ & $28.1^{b)}$ & 28.5 & 28.5 & 28.7 & $28.6^{h)}$ & $28.3^{j)}$ & $28.2^{m)}$ & 28.6 & 14 & 27.3 \\
\hline 3 & $28.8^{b)}$ & $29.3^{e)}$ & $29.3^{f)}$ & $29.4^{g)}$ & $28.9^{h)}$ & $28.8^{j)}$ & $28.7^{m)}$ & $26.8^{p)}$ & 15 & $29.4^{r)}$ \\
\hline $4^{\prime}$ & $28.9^{b)}$ & $29.3^{e)}$ & $29.3^{f)}$ & $29.4^{g)}$ & $29.4^{h)}$ & $29.1^{j)}$ & $29.1^{m)}$ & $130.7^{q)}$ & 16 & 31.6 \\
\hline $5^{\prime}$ & $23.9^{b)}$ & $29.3^{e)}$ & $29.3^{f)}$ & $29.4^{g)}$ & 27.0 & $29.1^{j)}$ & $26.9^{n)}$ & $127.4^{q)}$ & 17 & 22.7 \\
\hline $6^{\prime}$ & $29.4^{b)}$ & $29.5^{e)}$ & $29.5^{f)}$ & $29.7^{g)}$ & $129.2^{i)}$ & $29.3^{j)}(129.1)$ & $129.3^{o)}$ & 25.8 & 18 & 14.1 \\
\hline $7^{\prime}$ & $26.6^{c)}$ & $29.6^{e)}$ & $29.6^{f)}$ & $29.7^{g)}$ & $130.2^{i)}$ & $29.3^{j)}(129.7)$ & $127.5^{\circ)}$ & $128.2^{q)}$ & & \\
\hline $8^{\prime}$ & $129.3^{d)}$ & $29.6^{e)}$ & $29.6^{f 1}$ & $29.7^{g)}$ & 27.0 & $29.6^{j)}$ & 25.5 & $130.1^{q)}$ & & \\
\hline $9^{\prime}$ & $129.7^{d)}$ & $29.6^{e)}$ & 31.9 & $29.7^{g)}$ & 32.0 & $26.9^{k)}$ & $128.2^{o)}$ & $27.4^{p)}$ & & \\
\hline $10^{\prime}$ & $26.9^{c)}$ & $23.6^{e)}$ & 22.7 & $29.7^{g)}$ & 22.4 & $129.7^{l)}$ & $130.1^{o)}$ & 29.4 & & \\
\hline $11^{\prime}$ & 31.7 & 31.9 & 14.1 & $29.7^{g)}$ & 14.0 & $130.3^{l)}$ & $27.0^{n)}$ & 31.6 & & \\
\hline $12^{\prime}$ & 22.1 & 22.7 & & $29.9^{g)}$ & & $27.0^{k)}$ & $29.1^{m)}$ & 22.6 & & \\
\hline $13^{\prime}$ & 13.8 & 14.1 & & 32.0 & & 31.8 & 31.3 & 14.1 & & \\
\hline $14^{\prime}$ & & & & 22.8 & & $22.5 \quad(22.2)$ & 22.8 & & & \\
\hline $15^{\prime}$ & & & & 14.2 & & 14.0 & 13.9 & & & \\
\hline
\end{tabular}

a) This signal was not observed. $b-s$ ) Assignments may be interchanged in each column.

This suggested that the double bonds of the side chain existed at the $4^{\prime}$ - and $7^{\prime}$-positions, and were $Z$-form, based on the ${ }^{13} \mathrm{C}$-NMR chemical shifts of allyl carbon $(\delta 26.8,25.8$ and 27.4).

The biological activities of these compounds will be reported in the future.

\section{Experimental}

All melting points were determined on a Yanagimoto micro melting point apparatus and are uncorrected. Optical rotations were determined with a JASCO DIP-140 digital polarimeter, IR spectra were run on a JASCO A202 grating infrared spectrometer and UV spectra on a Shimadzu UV-360 spectrometer. MS were recorded on JEOL JMS D-100 instruments. Circular dichroism (CD) spectra were measured on a JASCO J-20A spectrometer. ${ }^{1} \mathrm{H}$ - and ${ }^{13} \mathrm{C}-\mathrm{NMR}$ spectra were recorded on a JEOL JNM-FX 90Q FT ( $90 \mathrm{MHz}$ and $22.5 \mathrm{MHz}$, respectively) and on a JEOL JNM-GX $400(399.5 \mathrm{MHz})$. Chemical shifts are given on the $\delta(\mathrm{ppm})$ scale with tetramethylsilane (TMS) as an internal standard (s, singlet; d, doublet; $t$, triplet; $m$, multiplet; br, broad). TLC was carried out on precoated Silica gel $60 \mathrm{~F}_{254}$ plates (Merck). Column chromatography was carried out on Silica gel type 60 (Merck). HPLC was run on JASCO 880-PC and UVIDEC-100V instruments.

Isolation - The dried fruits of E. rutaecarpa $(10 \mathrm{~kg})$ were extracted twice with $\mathrm{MeOH}$ under reflux to give the $\mathrm{MeOH}$ extract $(1.4 \mathrm{~kg})$, which was partitioned between AcOEt and water to give an AcOEt-soluble fraction $(550 \mathrm{~g})$. The AcOEt-soluble fraction was partitionated between benzene and water to give the benzene-soluble fraction $(280 \mathrm{~g})$. The benzene-soluble fraction was absorbed on Celite and eluted with $\mathrm{MeOH}-\mathrm{H}_{2} \mathrm{O}(50: 50)$ to give eluate 1 and with $\mathrm{MeOH}-\mathrm{H}_{2} \mathrm{O}(75: 25)$ to give eluate 2. Eluate 1 was passed through a Mitsubishi Diaion HP-20 column, and the absorbed material was eluted with $\mathrm{MeOH}-\mathrm{H}_{2} \mathrm{O}(75: 25)$ to give fraction 1 . Fraction 1 was chromatographed on a silica gel column [solvent, benzene-AcOEt $(85: 15)$ ] to give a limonoid mixture. The mixture was purified by HPLC [column, YMC-Pack D-ODS-7; $\mathrm{CH}_{3} \mathrm{CN}-\mathrm{H}_{2} \mathrm{O}(50: 50)$ ] to give compounds I-XI. Eluate 2 was concentrated under reduced pressure and the residue was chromatographed on a silica gel column [solvent, benzene-acetone $(85: 15)]$ to 
give a quinolone alkaloid mixture. The mixture was purified by HPLC [column, Nomura Chemical Develosil C8-10; $\left.\mathrm{MeOH}-\mathrm{H}_{2} \mathrm{O}(90: 10)\right]$ to give compounds XII-XIX.

Limonin (I) Colorless crystals $(45 \mathrm{mg}), \mathrm{mp} 271-275^{\circ} \mathrm{C}$ (AcOEt). Anal. Calcd for $\mathrm{C}_{26} \mathrm{H}_{30} \mathrm{O}_{8}$ : C, 66.37; $\mathrm{H}$, 6.43. Found: $\mathrm{C}, 66.65 ; \mathrm{H}, 6.48 .[\alpha]_{\mathrm{D}}^{20}-127.3^{\circ}\left(c=1.21\right.$, acetone). IR $v_{\max }^{\mathrm{KBr}} \mathrm{cm}^{-1}: 1765,1715,1315,1290,1270,1170$, $1035,1020,920,895,880,740,620,600 . \mathrm{UV} \lambda_{\max }^{\mathrm{MeOH}} \mathrm{nm}(\log \varepsilon): 208(3.77)$. MS m/z: $470(\mathrm{M})^{+}, 455\left(\mathrm{M}-\mathrm{CH}_{3}\right)^{+}, 347$ $\left(\mathrm{M}-\mathrm{C}_{6} \mathrm{H}_{3} \mathrm{O}_{3}\right)^{+} . \mathrm{CD}(c=0.011, \mathrm{MeOH})[\theta](\mathrm{nm}):-16000(228),-9300(291) .{ }^{1} \mathrm{H}-$ and ${ }^{13} \mathrm{C}-\mathrm{NMR}$ : Tables I and II

Rutaevine (II)-Colorless needles $(26 \mathrm{mg}), \mathrm{mp} 274-276^{\circ} \mathrm{C}\left(\mathrm{CHCl}_{3}-\mathrm{AcOEt}\right)$. Anal. Calcd for $\mathrm{C}_{26} \mathrm{H}_{30} \mathrm{O}_{9}: \mathrm{C}$, 64.19; H, 6.21. Found: C, 64.38; H, 6.26. $[\alpha]_{\mathrm{D}}^{20}-132.0^{\circ}\left(c=0.50, \mathrm{CH}_{3} \mathrm{CN}\right)$. IR $v_{\max }^{\mathrm{KBr}} \mathrm{cm}^{-1}: 1775,1745,1715,1390$, $1290,1180,1110,1060,1030,1005,955,905,880,825,730,700,600$. UV $\lambda_{\max }^{\mathrm{MeOH}} \mathrm{nm}(\log \varepsilon): 206(3.78)$. MS $m / z: 486$ $(\mathrm{M})^{+}, 471\left(\mathrm{M}-\mathrm{CH}_{3}\right)^{+}, 363\left(\mathrm{M}-\mathrm{C}_{6} \mathrm{H}_{3} \mathrm{O}_{3}\right)^{+} . \mathrm{CD}(c=0.006, \mathrm{MeOH})[\theta](\mathrm{nm}):-13000(221),-1900(298) .{ }^{1} \mathrm{H}-$ and ${ }^{13} \mathrm{C}$-NMR: Tables I and II.

Evodol (III)-Colorless powder $(11 \mathrm{mg}) \cdot[\alpha]_{\mathrm{D}}^{20}-156.4^{\circ}\left[c=1.04, \mathrm{CHCl}_{3}-\mathrm{MeOH}(1: 1)\right] . \mathrm{IR} v_{\max }^{\mathrm{KBr}} \mathrm{cm}^{-1}: 1750$, $1690,1660,1360,1290,1050,1030,915,875,600 . \mathrm{UV} \lambda_{\max }^{\mathrm{MeOH}} \mathrm{nm}(\log \varepsilon): 206(3.82), 276(3.95) . \mathrm{MS} m / z: 484(\mathrm{M})^{+}, 469$ $\left(\mathrm{M}-\mathrm{CH}_{3}\right)^{+}, 361\left(\mathrm{M}-\mathrm{C}_{6} \mathrm{H}_{3} \mathrm{O}_{3}\right)^{+} . \mathrm{CD}(c=0.005, \mathrm{MeOH})[\theta](\mathrm{nm}):-15000(230),+62000(275),-48000(319) .{ }^{1} \mathrm{H}-$ and ${ }^{13} \mathrm{C}$-NMR: Tables I and II.

Obacunone (IV)-Colorless rods $(14 \mathrm{mg}), \mathrm{mp} 216-222^{\circ} \mathrm{C}(\mathrm{EtOH}) .[\alpha]_{\mathrm{D}}^{20}-48.0^{\circ}\left(c=0.417, \mathrm{CHCl}_{3}\right) . \mathrm{IR} v_{\max }^{\mathrm{KBr}}$ $\mathrm{cm}^{-1}: 1750,1710,1400,1290,1170,1125,1080,1035,1000,930,890,835,815,615 . \mathrm{MS} \mathrm{m} / \mathrm{z}: 454(\mathrm{M})^{+}, 439$ $\left(\mathrm{M}-\mathrm{CH}_{3}\right)^{+}, 331\left(\mathrm{M}-\mathrm{C}_{6} \mathrm{H}_{3} \mathrm{O}_{3}\right)^{+} . \mathrm{CD}(c=0.005, \mathrm{MeOH})[\theta](\mathrm{nm}):+11000(220),-12700(247),-9000(288) .{ }^{1} \mathrm{H}-$ and ${ }^{13} \mathrm{C}-\mathrm{NMR}$ : Tables I and II.

Jangomolide (V)-Colorless crystals $(30 \mathrm{mg})$, mp 258.5-259.5 ${ }^{\circ} \mathrm{C}\left(\mathrm{CHCl}_{3}-\mathrm{EtOH}\right)$. Anal. Calcd for $\mathrm{C}_{26} \mathrm{H}_{28} \mathrm{O}_{8}$ : $\mathrm{C}, 65.61 ; \mathrm{H}, 6.29$. Found: $\mathrm{C}, 65.62 ; \mathrm{H}, 6.07 .[\alpha]_{\mathrm{D}}^{20}+20.3^{\circ}\left[c=0.62, \mathrm{CHCl}_{3}-\mathrm{MeOH}(1: 1)\right] . \mathrm{IR} v_{\max }^{\mathrm{KBr}} \mathrm{cm}^{-1}: 1720,1385$, $1345,1285,1270,1060,1020,980,920,875,810,600$. UV $\lambda_{\max }^{\mathrm{MeOH}} \mathrm{nm}(\log \varepsilon): 209(4.20) . \mathrm{MS} m / z: 468(\mathrm{M})^{+}, 453$ $\left(\mathrm{M}-\mathrm{CH}_{3}\right)^{+}, 345\left(\mathrm{M}-\mathrm{C}_{6} \mathrm{H}_{3} \mathrm{O}_{3}\right)^{+} . \mathrm{CD}(c=0.005, \mathrm{MeOH})[\theta](\mathrm{nm}):-39000(220),+7000(303) .{ }^{1} \mathrm{H}-$ and ${ }^{13} \mathrm{C}-\mathrm{NMR}$ : Tables I and II.

Rutaevine Acetate (VI)-Colorless crystals $(43 \mathrm{mg}), \mathrm{mp} 183-186^{\circ} \mathrm{C}(\mathrm{EtOH}) .[\alpha]_{\mathrm{D}}^{20}-104.0^{\circ}(c=1.00$, $\left.\mathrm{CH}_{3} \mathrm{CN}\right)$. IR $v_{\max }^{\mathrm{KBr}} \mathrm{cm}^{-1}: 1760,1720,1395,1375,1290,1230,1180,1110,1060,1030,1010,960,910,880,770,700,605$. $\mathrm{UV} \lambda_{\max }^{\mathrm{MeOH}} \mathrm{nm}(\log \varepsilon): 206$ (3.81). $\mathrm{MS} m / z: 528(\mathrm{M})^{+}, 513\left(\mathrm{M}-\mathrm{CH}_{3}\right)^{+}, 468\left(\mathrm{M}-\mathrm{CH}_{3} \mathrm{COOH}\right)^{+}, 363$ $\left(\mathrm{M}-\mathrm{C}_{6} \mathrm{H}_{3} \mathrm{O}_{3}-\mathrm{CH}_{2} \mathrm{CO}\right)^{+} . \mathrm{CD}(c=0.006, \mathrm{MeOH})[\theta](\mathrm{nm}):-21000(224),-1700(290) .{ }^{1} \mathrm{H}-$ and ${ }^{13} \mathrm{C}-\mathrm{NMR}$ : Tables I and II.

Graucin A (VII)-Colorless crystals $(30 \mathrm{mg}), \mathrm{mp} 314-316^{\circ} \mathrm{C}\left(\mathrm{CH}_{3} \mathrm{CN}\right) .[\alpha]_{\mathrm{D}}^{20}-110.0^{\circ}\left(c=0.20, \mathrm{CH}_{3} \mathrm{CN}\right) . \mathrm{IR}$ $v_{\max }^{\mathrm{KBr}} \mathrm{cm}^{-1}: 3475,1775,1750,1705,1405,1375,1300,1185,1170,1110,1075,1055,1015,960,880,820,775,760,610$. $\mathrm{UV} \lambda_{\max }^{\mathrm{MeOH}} \mathrm{nm}(\log \varepsilon): 206(3.55)$. MS $m / z: 502(\mathrm{M})^{+}, 487\left(\mathrm{M}-\mathrm{CH}_{3}\right)^{+}, 379\left(\mathrm{M}-\mathrm{C}_{6} \mathrm{H}_{3} \mathrm{O}_{3}\right)^{+} . \mathrm{CD}(c=0.007, \mathrm{MeOH})[\theta]$ (nm): -9100 (224), -1500 (290). ${ }^{1} \mathrm{H}$ - and ${ }^{13} \mathrm{C}-\mathrm{NMR}$ : Tables I and II.

$12 \alpha$-Hydroxylimonin (VIII) Colorless powder $(27 \mathrm{mg}),[\alpha]_{\mathrm{D}}^{20}-134.0^{\circ}\left(c=1.0, \mathrm{CHCl}_{3}\right) . \mathrm{IR} v_{\max }^{\mathrm{KBr}} \mathrm{cm}^{-1}: 3475$, $1750,1720,1285,1165,1045,1020,940,910,900,875,810,620,600,580$. UV $\lambda_{\max }^{\mathrm{MeOH}} \mathrm{nm}(\log \varepsilon): 205(3.76)$. MS $m / z$ : $485(\mathrm{M}-\mathrm{H})^{+}, 471\left(\mathrm{M}-\mathrm{CH}_{3}\right)^{+}, 363\left(\mathrm{M}-\mathrm{C}_{6} \mathrm{H}_{3} \mathrm{O}_{3}\right)^{+} . \mathrm{CD}(c=0.005, \mathrm{MeOH})[\theta](\mathrm{nm}):-12200(227),-6300(290)$. ${ }^{1} \mathrm{H}$ - and ${ }^{13} \mathrm{C}$-NMR: Tables I and II.

$12 \alpha$-Hydroxyevodol (IX)-Amorphous solid $(6 \mathrm{mg}),[\alpha]_{\mathrm{D}}^{20}-114.0^{\circ}(c=0.48, \mathrm{MeOH}) . \mathrm{IR} v_{\max }^{\mathrm{KBr}} \mathrm{cm}^{-1}: 3450$, $1750,1690,1385,1355,1285,1050,1030,880,600$. UV $\lambda_{\max }^{\mathrm{MeOH}} \mathrm{nm}(\log \varepsilon): 205(3.84), 276(3.67) . \mathrm{MS} m / z: 500(\mathrm{M})^{+}$, $485\left(\mathrm{M}-\mathrm{CH}_{3}\right)^{+} . \mathrm{CD}(c=0.005, \mathrm{MeOH})[\theta](\mathrm{nm}):-8800(228),+27800(275),-22200(320) .{ }^{1} \mathrm{H}-$ and ${ }^{13} \mathrm{C}-\mathrm{NMR}$ : Tables I and II.

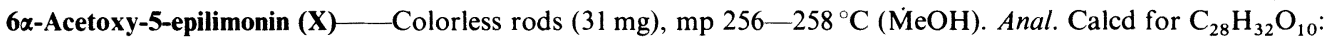
$\mathrm{C}, 63.64 ; \mathrm{H}, 6.10$. Found: C, 63.49: H, 6.14. $[\alpha]_{\mathrm{D}}^{20}-93.5^{\circ}\left(c=1.0, \mathrm{CHCl}_{3}\right)$. IR $v_{\max }^{\mathrm{KBr}} \mathrm{cm}^{-1}: 1765,1750,1740,1380,1295$, $1250,1180,1060,1025,1005,980,915,880,810,605$. UV $\lambda_{\max }^{\mathrm{MeOH}} \mathrm{nm}(\log \varepsilon): 208(3.76) . \mathrm{MS} m / z: 528(\mathrm{M})^{+}, 513$ $\left(\mathrm{M}-\mathrm{CH}_{3}\right)^{+}, 453\left(\mathrm{M}-\mathrm{CH}_{3} \mathrm{COOH}\right)^{+}, 387\left(\mathrm{M}-\mathrm{C}_{6} \mathrm{H}_{3} \mathrm{O}_{3}\right)^{+} . \mathrm{CD}(c=0.007, \mathrm{MeOH})[\theta](\mathrm{nm}):-19000(225),-4000$ (287). ${ }^{1} \mathrm{H}-\mathrm{NMR}\left(\mathrm{CDCl}_{3}\right)(400 \mathrm{MHz}) \delta: 1.14\left(3 \mathrm{H}, \mathrm{s}, 18-\mathrm{H}_{3}\right), 1.22\left(3 \mathrm{H}, \mathrm{s}, 30-\mathrm{H}_{3}\right), 1.27\left(3 \mathrm{H}, \mathrm{s}, 28-\mathrm{H}_{3}\right), 1.37(3 \mathrm{H}, \mathrm{s}, 29-$ $\left.\mathrm{H}_{3}\right), 1.48\left(1 \mathrm{H}, \mathrm{ddd}, J=15.5,7,3.5 \mathrm{~Hz}, 12-\mathrm{H}_{\alpha}\right), 1.70\left(1 \mathrm{H}\right.$, dddd, $\left.J=15.5,7,4,2 \mathrm{~Hz}, 11-\mathrm{H}_{\alpha}\right), 1.87(1 \mathrm{H}, \mathrm{dddd}, J=15.5$, $\left.12,7.5,3.5 \mathrm{~Hz}, 11-\mathrm{H}_{\beta}\right), 1.88\left(1 \mathrm{H}, \mathrm{ddd}, J=15.5,7.5,4 \mathrm{~Hz}, 12-\mathrm{H}_{\beta}\right), 2.21\left(3 \mathrm{H}, \mathrm{s}, \mathrm{CH}_{3} \mathrm{CO}\right), 2.61(1 \mathrm{H}, \mathrm{dd}, J=15.5,3.5 \mathrm{~Hz}$, $\left.2-\mathrm{H}_{\alpha}\right), 2.85\left(1 \mathrm{H}, \mathrm{dd}, J=15.5,2.5 \mathrm{~Hz}, 2-\mathrm{H}_{\beta}\right), 2.92(1 \mathrm{H}, \mathrm{dd}, J=12,2 \mathrm{~Hz}, 9-\mathrm{H}), 3.08(1 \mathrm{H}, \mathrm{dd}, J=3.5,3.2 \mathrm{~Hz}, 1-\mathrm{H}), 4.23$ $\left(1 \mathrm{H}, \mathrm{d}, J=12 \mathrm{~Hz}, 19-\mathrm{H}_{\alpha}\right), 4.64\left(1 \mathrm{H}, \mathrm{d}, J=12 \mathrm{~Hz}, 19-\mathrm{H}_{\beta}\right), 5.44(1 \mathrm{H}, \mathrm{s}, 17-\mathrm{H}), 6.05(1 \mathrm{H}, \mathrm{d}, J=10 \mathrm{~Hz}, 6-\mathrm{H}), 6.35(1 \mathrm{H}, \mathrm{dd}$, $j=1.8,1 \mathrm{~Hz}, 22-\mathrm{H}), 7.40(1 \mathrm{H}, \mathrm{t}, J=1.8 \mathrm{~Hz}, 23-\mathrm{H}), 7.42(1 \mathrm{H}, \mathrm{dd}, J=1.8,1 \mathrm{~Hz}, 21-\mathrm{H}) .{ }^{13} \mathrm{C}-\mathrm{NMR}$ : See the previous paper.9)

6ק-Acetoxy-5-epilimonin (XI) Colorless crystals $(36 \mathrm{mg})$, $\dot{\mathrm{mp}} 229-231^{\circ} \mathrm{C}(\mathrm{MeOH})$. Anal. Calcd for $\mathrm{C}_{28} \mathrm{H}_{32} \mathrm{O}_{10}: \mathrm{C}, 63.64 ; \mathrm{H}, 6.10$. Found: $\mathrm{C}, 63.37 ; \mathrm{H}, 6.09$. $[\alpha]_{\mathrm{D}}^{20}+4.1 .0^{\circ}\left(c=1.0, \mathrm{CHCl}_{3}\right)$. IR $v_{\max }^{\mathrm{KBr}} \mathrm{cm}^{-1}: 1770,1750,1720$, $1385,1285,1240,1155,1055,1020,980,880,805,600$. UV $\lambda_{\max }^{\mathrm{MeOH}} \mathrm{nm}(\log \varepsilon): 206(3.78) . \mathrm{MS} m / z: 513\left(\mathrm{M}_{-}-\mathrm{CH}_{3}\right)^{+}, 453$ $\left(\mathrm{M}-\mathrm{CH}_{3}-\mathrm{CH}_{3} \mathrm{COOH}\right)^{+} . \mathrm{CD}(c=0.010, \mathrm{MeOH})[\theta](\mathrm{nm}):-11500(230),+12000(295),+11000(303) .{ }^{1} \mathrm{H}-$ and ${ }^{13} \mathrm{C}-\mathrm{NMR}$ : See the previous paper. ${ }^{9)}$

Acetylation of II-Acetic anhydride ( 2 drops) was added to a solution of II (11 mg) in pyridine $(0.5 \mathrm{ml})$, and the reaction mixture was left for $3 \mathrm{~h}$ at room temperature. After evaporation of the solvent, the product was purified by HPLC using a reversed-phase column to give the acetate (II-Ac) as colorless crystals ( $9 \mathrm{mg}$ ). II-Ac was identical 
with VI by ${ }^{1} \mathrm{H}-\mathrm{NMR}$ spectral comparison.

Acetylation of III-III (4 mg) was acetylated in the same way as II, giving the acetate (III-Ac) as colorless crystals $(4 \mathrm{mg}) .{ }^{1} \mathrm{H}-\mathrm{NMR}\left(\mathrm{CDCl}_{3}\right) \delta: 1.00,1.16,1.46,1.46\left(3 \mathrm{H}, \mathrm{s}, 18-, 28-, 29-, 30-\mathrm{H}_{3}\right), 2.28(3 \mathrm{H}, \mathrm{s}, 6-\mathrm{OAc}), 4.06(1 \mathrm{H}$, s, $15-\mathrm{H}), 4.18(1 \mathrm{H}$, br t, $J=2.5 \mathrm{~Hz}, 1-\mathrm{H}), 4.70\left(2 \mathrm{H}, \mathrm{s}, 19-\mathrm{H}_{2}\right), 5.46(1 \mathrm{H}, \mathrm{s}, 17-\mathrm{H}), 6.38(1 \mathrm{H}, \mathrm{br} \mathrm{s}, 22-\mathrm{H}), 7.45(2 \mathrm{H}, \mathrm{br} \mathrm{s}$, $21-, 23-\mathrm{H})$.

Acetylation of VII_ _ VII (10 mg) was acetylated in the same way as II, giving the acetate (VII-Ac) as colorless crystals $(8 \mathrm{mg}) .{ }^{1} \mathrm{H}-\mathrm{NMR}\left(\mathrm{CDCl}_{3}\right) \delta: 0.79,1.33,1.42,1.42\left(3 \mathrm{H}, \mathrm{s}, 18-, 28-, 29-, 30-\mathrm{H}_{3}\right), 1.83(3 \mathrm{H}, \mathrm{s}, 12-\mathrm{OAc}), 2.30(3 \mathrm{H}$, s, 7-OAc), $2.56\left(1 \mathrm{H}, \mathrm{dd}, J=16,3 \mathrm{~Hz}, 2-\mathrm{H}_{\alpha}\right), 2.90\left(1 \mathrm{H}, \mathrm{dd}, J=16,2.5 \mathrm{~Hz}, 2-\mathrm{H}_{\beta}\right), 3.09(1 \mathrm{H}, \mathrm{brd}, J=11 \mathrm{~Hz}, 9-\mathrm{H}), 3.12$ $(1 \mathrm{H}, \mathrm{s}, 5-\mathrm{H}), 3.84(1 \mathrm{H}, \mathrm{s}, 15-\mathrm{H}), 4.06\left(1 \mathrm{H}, \mathrm{d}, J=13 \mathrm{~Hz}, 19-\mathrm{H}_{\alpha}\right), 4.24\left(1 \mathrm{H}, \mathrm{d}, J=13 \mathrm{~Hz}, 19-\mathrm{H}_{\beta}\right), 5.02(1 \mathrm{H}, \mathrm{d}, J=5 \mathrm{~Hz}$, $12-\mathrm{H}), 5.56(1 \mathrm{H}, \mathrm{s}, 17-\mathrm{H}), 5.67(1 \mathrm{H}, \mathrm{s}, 7-\mathrm{H}), 6.39(1 \mathrm{H}$, br s, 22-H), 7.46, $7.48(1 \mathrm{H}$, br s, 21-, 23-H).

Acetylation of VIII-VIII $(10 \mathrm{mg})$ was acetylated in the same way as II, giving the acetate as a colorless powder (VIII-Ac) $(4.5 \mathrm{mg}) .{ }^{1} \mathrm{H}-\mathrm{NMR}\left(\mathrm{CDCl}_{3}\right) \delta: 1.01,1.18,1.31,1.31\left(3 \mathrm{H}, \mathrm{s}, 18-, 28-, 29-, 30-\mathrm{H}_{3}\right), 1.83(3 \mathrm{H}, \mathrm{s}, 12-\mathrm{OAc}), 4.86$ $(1 \mathrm{H}, \mathrm{d}, J=5 \mathrm{~Hz}, 12-\mathrm{H}), 5.50(1 \mathrm{H}, \mathrm{s}, 17-\mathrm{H}), 6.40(1 \mathrm{H}, \mathrm{br} \mathrm{s}, 22-\mathrm{H}), 7.43(2 \mathrm{H}$, br s, $21-, 23-\mathrm{H})$.

Acetylation of IX $\mathrm{IX}(3 \mathrm{mg})$ was acetylated in the same way as II, giving the acetate (IX-Ac) as a colorless powder $(2.5 \mathrm{mg}) .{ }^{1} \mathrm{H}-\mathrm{NMR}\left(\mathrm{CDCl}_{3}\right) \delta: 1.06,1.20,1.45,1.49\left(3 \mathrm{H}, \mathrm{s}, 18-, 28-, 29-, 30-\mathrm{H}_{3}\right), 1.74(3 \mathrm{H}, \mathrm{s}, 12-\mathrm{OAc}), 2.28$ $(3 \mathrm{H}, \mathrm{s}, 6-\mathrm{OAc}), 4.12(1 \mathrm{H}, \mathrm{s}, 15-\mathrm{H}), 4.42\left(1 \mathrm{H}, \mathrm{d}, J=13 \mathrm{~Hz}, 19-\mathrm{H}_{\alpha}\right), 4.86\left(1 \mathrm{H}, \mathrm{d}, J=13 \mathrm{~Hz}, 19-\mathrm{H}_{\beta}\right), 5.02(1 \mathrm{H}, \mathrm{d}, J=5 \mathrm{~Hz}$, 12-H), $5.46(1 \mathrm{H}, \mathrm{s}, 17-\mathrm{H}), 6.38(1 \mathrm{H}$, br s, 22-H), 7.42, $7.44(1 \mathrm{H}$, br s, 21-, 23-H).

Alkali Hydrolysis of $\mathbf{X}$-Aqueous $0.1 \mathrm{~N} \mathrm{KHCO}_{3}(0.5 \mathrm{ml})$ was added to a solution of $\mathrm{X}(5 \mathrm{mg})$ in $\mathrm{MeOH}$ $(4.5 \mathrm{ml})$. The mixture was stirred for $24 \mathrm{~h}$ at room temperature. After being diluted with water, the mixture was passed through a Mitsubishi Diaion HP-20 column, which was washed with water, then eluted with $\mathrm{MeOH}$. The $\mathrm{MeOH}$ eluate was purified by HPLC using a reversed-phase column to give a colorless amorphous product (X-C) $(0.31 \mathrm{mg})$. $\mathrm{X}-\mathrm{C}$ was identical with III by ${ }^{1} \mathrm{H}-\mathrm{NMR}$ spectral comparison.

Alkali Hydrolysis of XI-XI ( $5 \mathrm{mg})$ was hydrolyzed in the same way as $\mathrm{X}$, giving a colorless amorphous product $(\mathrm{X}-\mathrm{IC})(0.25 \mathrm{mg})$, which was identical with III by ${ }^{1} \mathrm{H}-\mathrm{NMR}$ spectral comparison.

Evocarpine (XII)-Colorless oil (457 mg). IR $v_{\max }^{\mathrm{KBr}} \mathrm{cm}^{-1}: 2960,2940,2860,1635,1605,1505,1475,1180,775$, 760. UV $\lambda_{\max }^{\mathrm{MeOH}} \mathrm{nm}(\log \varepsilon): 213(4.41), 239(4.47), 321(4.14), 333(4.15) . \mathrm{MS} m / z: 339(\mathrm{M})^{+}(26), 186\left(\mathrm{M}-\mathrm{C}_{11} \mathrm{H}_{21}\right)^{+}$ (100), $173\left(\mathrm{M}-\mathrm{C}_{12} \mathrm{H}_{22}\right)^{+}(93), 144\left(\mathrm{M}-\mathrm{C}_{13} \mathrm{H}_{23} \mathrm{O}\right)^{+}(12) .{ }^{1} \mathrm{H}-\mathrm{NMR}\left(\mathrm{CDCl}_{3}\right) \delta: 0.90\left(3 \mathrm{H}, \mathrm{br} \mathrm{t}, J=6 \mathrm{~Hz}, 13^{\prime}-\mathrm{H}_{3}\right), 1.35$ $\left(12 \mathrm{H}, \mathrm{m}, 2^{\prime}-, 3^{\prime}-, 4^{\prime}-, 5^{\prime}-, 6^{\prime}-, 11^{\prime}-\mathrm{H}_{2}\right), 1.70\left(2 \mathrm{H}, \mathrm{m}, 12^{\prime}-\mathrm{H}_{2}\right), 2.72\left(2 \mathrm{H}, \mathrm{brt}, J=8 \mathrm{~Hz}, \mathrm{l}^{\prime}-\mathrm{H}\right), 3.76\left(3 \mathrm{H}, \mathrm{s}, \mathrm{N}-\mathrm{CH}_{3}\right), 5.40$ $\left(2 \mathrm{H}\right.$, br t $\left., J=5 \mathrm{~Hz}, 8^{\prime}-, 9^{\prime}-\mathrm{H}\right), 6.26(1 \mathrm{H}, \mathrm{s}, 3-\mathrm{H}), 7.3-7.8(3 \mathrm{H}, \mathrm{m}, 6-, 7-, 8-\mathrm{H}), 8.50(1 \mathrm{H}, \mathrm{dd}, J=8,2 \mathrm{~Hz}, 5-\mathrm{H}) .{ }^{13} \mathrm{C}-$ NMR: Table III.

Dihydroevocarpine (XIII) C Colorless powder $(21.8 \mathrm{mg}), \mathrm{mp} 74.5-75.5^{\circ} \mathrm{C}$ (AcOEt). IR $v_{\max }^{\mathrm{KBr}} \mathrm{cm}^{-1}: 2960,2930$, $2860,1645,1605,1575,1500,1475,1180,775,760$. UV $\lambda_{\max }^{\mathrm{MeOH}} \mathrm{nm}(\log \varepsilon): 213(4.40), 239$ (4.46), 321 (4.13), 333 (4.15). MS $m / z: 341(\mathrm{M})^{+}(12), 186\left(\mathrm{M}-\mathrm{C}_{11} \mathrm{H}_{23}\right)^{+}(100), 173\left(\mathrm{M}-\mathrm{C}_{12} \mathrm{H}_{24}\right)^{+}(96), 144\left(\mathrm{M}-\mathrm{C}_{13} \mathrm{H}_{25} \mathrm{O}\right)^{+}(16) .{ }^{1} \mathrm{H}-\mathrm{NMR}$ $\left(\mathrm{CDCl}_{3}\right) \delta: 0.90\left(3 \mathrm{H}\right.$, br t, $\left.J=6 \mathrm{~Hz}, 13^{\prime}-\mathrm{H}_{3}\right), 1.27\left(20 \mathrm{H}\right.$, br s, $\left.2^{\prime}-, 3^{\prime}-, 4^{\prime}-, 5^{\prime}-, 6^{\prime}-, 7^{\prime}-, 8^{\prime}-, 9^{\prime}-, 10^{\prime}-, 11^{\prime}-\mathrm{H}_{2}\right), 1.60(2 \mathrm{H}$, br m, 12'- $\left.\mathrm{H}_{2}\right), 2.70\left(2 \mathrm{H}\right.$, br t, $\left.J=8 \mathrm{~Hz}, 1^{\prime}-\mathrm{H}_{2}\right), 3.76\left(3 \mathrm{H}, \mathrm{s}, \mathrm{N}-\mathrm{CH}_{3}\right), 6.24(1 \mathrm{H}, \mathrm{s}, 3-\mathrm{H}), 7.3-7.8(3 \mathrm{H}, \mathrm{m}, 6-, 7-, 8-\mathrm{H})$, $8.50(1 \mathrm{H}, \mathrm{dd}, J=8,2 \mathrm{~Hz}, 5-\mathrm{H}) .{ }^{13} \mathrm{C}-\mathrm{NMR}$ : Table III.

1-Methyl-2-undecyl-4(1 H)-quinolone (XIV) Colorless powder $(19 \mathrm{mg}), \mathrm{mp} 69-70^{\circ} \mathrm{C}$ (AcOEt-ether). IR $v_{\max }^{\mathrm{KBr}}$ $\mathrm{cm}^{-1}: 2960,2940,2860,1645,1600,1575,1500,1475,1180,775,760$. UV $\lambda_{\max }^{\mathrm{MeOH}} \mathrm{nm}(\log \varepsilon): 213(4.40), 239(4.46), 321$ (4.13), $333(4.15)$. MS $m / z: 313(\mathrm{M})^{+}(20), 186\left(\mathrm{M}-\mathrm{C}_{9} \mathrm{H}_{19}\right)^{+}(100), 173\left(\mathrm{M}-\mathrm{C}_{10} \mathrm{H}_{20}\right)^{+}(100), 144\left(\mathrm{M}-\mathrm{C}_{11} \mathrm{H}_{21} \mathrm{O}\right)^{+}$ (16). ${ }^{1} \mathrm{H}-\mathrm{NMR}\left(\mathrm{CDCl}_{3}\right) \delta: 0.90\left(3 \mathrm{H}\right.$, br t, $\left.J=6 \mathrm{~Hz}, 11^{\prime}-\mathrm{H}_{3}\right), 1.27\left(18 \mathrm{H}\right.$, br s, 2'-, 3'-, 4'-, 5'-, 6'-, 7'-, 8'-, 9'-, 10' ' $\left.^{\prime} \mathrm{H}_{2}\right)$, $2.73\left(2 \mathrm{H}, \mathrm{brt}, J=8 \mathrm{~Hz}, \mathrm{l}^{\prime}-\mathrm{H}_{2}\right), 3.78\left(3 \mathrm{H}, \mathrm{s}, \mathrm{N}-\mathrm{CH}_{3}\right), 6.28(1 \mathrm{H}, \mathrm{s}, 3-\mathrm{H}), 7.3-7.8(3 \mathrm{H}, \mathrm{m}, 6-, 7-, 8-\mathrm{H}), 8.50(1 \mathrm{H}, \mathrm{dd}, J=$ $8,2 \mathrm{~Hz}, 5-\mathrm{H}) .{ }^{13} \mathrm{C}-\mathrm{NMR}$ : Table III.

1-Methyl-2-pentadecyl-4(1 H)-quinolone $(\mathrm{XV})$ - Colorless powder $(7 \mathrm{mg}), \mathrm{mp} 65-66^{\circ} \mathrm{C}$ (AcOEt-ether). IR $v_{\max }^{\mathrm{KBr}} \mathrm{cm}^{-1}: 2960,2930,2860,1645,1605,1575,1505,1475,1180,775,760$. UV $\lambda_{\max }^{\mathrm{MeOH}} \mathrm{nm}(\log \varepsilon): 213(4.40), 239(4.46)$, 321 (4.13), 333 (4.15). MS m/z: $369(\mathrm{M})^{+}(24), 186\left(\mathrm{M}-\mathrm{C}_{13} \mathrm{H}_{27}\right)^{+}(100), 173\left(\mathrm{M}-\mathrm{C}_{14} \mathrm{H}_{28}\right)^{+}(93), 144$ $\left(\mathrm{M}-\mathrm{C}_{15} \mathrm{H}_{29} \mathrm{O}\right)^{+}(5) .{ }^{1} \mathrm{H}-\mathrm{NMR}\left(\mathrm{CDCl}_{3}\right) \delta: 0.90\left(3 \mathrm{H}\right.$, br t, $\left.J=6 \mathrm{~Hz}, 15^{\prime}-\mathrm{H}_{3}\right), 1.28\left(24 \mathrm{H}\right.$, br s, $2^{\prime}-, 3^{\prime}-, 4^{\prime}-, 5^{\prime}-, 6^{\prime}-, 7^{\prime}-$, $\left.8^{\prime}-, 9^{\prime}-, 10^{\prime}-11^{\prime}-, 12^{\prime}-, 13^{\prime}-\mathrm{H}_{2}\right), 1.64\left(2 \mathrm{H}\right.$, br m, 14' $\left.-\mathrm{H}_{2}\right), 2.76\left(2 \mathrm{H}\right.$, brt, $\left.J=8 \mathrm{~Hz}, 1^{\prime}-\mathrm{H}_{2}\right), 3.79\left(3 \mathrm{H}, \mathrm{s}, \mathrm{N}-\mathrm{CH}_{3}\right), 6.26$ $(1 \mathrm{H}, \mathrm{s}, 3-\mathrm{H}), 7.3-7.8(3 \mathrm{H}, \mathrm{m}, 6-, 7-, 8-\mathrm{H}), 8.50(1 \mathrm{H}, \mathrm{dd}, J=8,2 \mathrm{~Hz}, 5-\mathrm{H}) .{ }^{13} \mathrm{C}-\mathrm{NMR}$ : Table III.

1-Methyl-2-[(Z)-6-undecenyl]-4(1 H)-quinolone (XVI) Colorless oil $(10 \mathrm{mg}) . \mathrm{IR} v_{\max }^{\mathrm{KBr}} \mathrm{cm}^{-1}: 2960,2940,2860$, $1635,1605,1575,1505,1470,1180,775,760 . \mathrm{UV} \lambda_{\max }^{\mathrm{MeOH}} \mathrm{nm}(\log \varepsilon): 213$ (4.43), 239 (4.49), 321 (4.16), 333 (4.17). MS $m / z: 311(\mathrm{M})^{+}(30), 186\left(\mathrm{M}-\mathrm{C}_{9} \mathrm{H}_{17}\right)^{+}(100), 173\left(\mathrm{M}-\mathrm{C}_{10} \mathrm{H}_{18}\right)^{+}(79), 144\left(\mathrm{M}-\mathrm{C}_{11} \mathrm{H}_{19} \mathrm{O}\right)^{+}(20) .{ }^{1} \mathrm{H}-\mathrm{NMR}\left(\mathrm{CDCl}_{3}\right)$ $\delta: 0.90\left(3 \mathrm{H}\right.$, br t, $\left.J=6 \mathrm{~Hz}, 11^{\prime}-\mathrm{H}_{3}\right), 1.2-1.8\left(10 \mathrm{H}\right.$, br m, 2'-, 3'-, 4'-, 9'-, 10' $\left.-\mathrm{H}_{2}\right), 1.9-2.3\left(4 \mathrm{H}\right.$, br m, $\left.5^{\prime}-, 8^{\prime}-\mathrm{H}_{2}\right), 2.71$ $\left(2 \mathrm{H}, \mathrm{brt}, J=8 \mathrm{~Hz}, \mathrm{l}^{\prime}-\mathrm{H}_{2}\right), 3.73\left(3 \mathrm{H}, \mathrm{s}, \mathrm{N}-\mathrm{CH}_{3}\right), 5.35\left(2 \mathrm{H}, \mathrm{m}, 6^{\prime}-7^{\prime}-\mathrm{H}\right), 6.22(1 \mathrm{H}, \mathrm{s}, 3-\mathrm{H}), 7.3-7.8(3 \mathrm{H}, \mathrm{m}, 6-, 7-, 8-$ H), $8.41(1 \mathrm{H}, \mathrm{dd}, J=8,2 \mathrm{~Hz}, 5-\mathrm{H}) \cdot{ }^{13} \mathrm{C}-\mathrm{NMR}$ : Table III.

Compound XVII \{1-Methyl-2-[(Z)-10-pentadecenyl]-4(1 H)-quinolone (XVIIa) + 1-Methyl-2-[( $Z$ )-6-pentadecenyl]-4(1 H)-quinolone (XVIIb) $\}$ Colorless oil $(47 \mathrm{mg}) . \mathrm{IR} v_{\max }^{\mathrm{KBr}} \mathrm{cm}^{-1}: 2960,2940,2860,1630,1605,1505,1470$, 1180, 760. UV $\lambda_{\max }^{\mathrm{MeOH}} \mathrm{nm}(\log \varepsilon): 214$ (4.40), 239 (4.47), 321 (4.13), 333 (4.14). MS m/z: $367(\mathrm{M})^{+}(30), 186$ $\left(\mathrm{M}-\mathrm{C}_{13} \mathrm{H}_{25}\right)^{+}(100), 173\left(\mathrm{M}-\mathrm{C}_{14} \mathrm{H}_{26}\right)^{+}(82), 144\left(\mathrm{M}-\mathrm{C}_{15} \mathrm{H}_{27} \mathrm{O}\right)^{+}(12) .{ }^{1} \mathrm{H}-\mathrm{NMR}\left(\mathrm{CDCl}_{3}\right) \delta: 0.90(3 \mathrm{H}$, br m, 15'$\left.\mathrm{H}_{3}\right), 1.32(18 \mathrm{H}, \mathrm{brm}), 2.70\left(2 \mathrm{H}\right.$, br t, $\left.J=8 \mathrm{~Hz}, 1^{\prime}-\mathrm{H}_{2}\right), 3.74\left(3 \mathrm{H}, \mathrm{s}, \mathrm{N}-\mathrm{CH}_{3}\right) ; 5.40(2 \mathrm{H}, \mathrm{brt}, J=5 \mathrm{~Hz}), 6.25(1 \mathrm{H}, \mathrm{s}, 3-\mathrm{H})$, 7.3-7.8 (3H, m, 6-, 7-, 8-H), $8.48(1 \mathrm{H}, \mathrm{dd}, J=8,2 \mathrm{~Hz}, 5-\mathrm{H}) .{ }^{13} \mathrm{C}-\mathrm{NMR}$ : Table III. 
1-Methyl-2-[(6Z,9Z)-6,9-pentadecadienyl]-4(1 H)-quinolone (XVIII)-Colorless oil (45 mg). IR $v_{\max }^{\mathrm{KBr}} \mathrm{cm}^{-1}$ : 2960, 2940, 2860, 1630, 1605, 1505, 1470, 1180, 760. UV $\lambda_{\max }^{\mathrm{MeOH}} \mathrm{nm}(\log \varepsilon): 214(4.41), 239(4.50), 321$ (4.11), 333 (4.13). MS $m / z: 365(\mathrm{M})^{+}(39), 186\left(\mathrm{M}-\mathrm{C}_{13} \mathrm{H}_{23}\right)^{+}(100), 173\left(\mathrm{M}-\mathrm{C}_{14} \mathrm{H}_{24}\right)^{+}(86), 144\left(\mathrm{M}-\mathrm{C}_{15} \mathrm{H}_{25} \mathrm{O}\right)^{+}(19) .{ }^{1} \mathrm{H}-\mathrm{NMR}$ $\left(\mathrm{CDCl}_{3}\right) \delta: 0.90\left(3 \mathrm{H}\right.$, br t, $\left.J=6 \mathrm{~Hz}, 15^{\prime}-\mathrm{H}_{3}\right), 1.40\left(12 \mathrm{H}\right.$, br m, 2'-, 3'-, 4'-, 12'-, 13'-, 14'- $\left.\mathrm{H}_{2}\right), 2.08(4 \mathrm{H}$, br m, 5'-, 11'$\left.\mathrm{H}_{2}\right), 2.70\left(4 \mathrm{H}, \mathrm{m}, \mathrm{l}^{\prime}-, 8^{\prime}-\mathrm{H}_{2}\right), 3.69\left(3 \mathrm{H}, \mathrm{s}, \mathrm{N}-\mathrm{CH}_{3}\right), 5.40\left(4 \mathrm{H}, \mathrm{m}, 6^{\prime}-, 7^{\prime}-, 9^{\prime}-, 0^{\prime}-\mathrm{H}\right), 6.15(1 \mathrm{H}, \mathrm{s}, 3-\mathrm{H}), 7.25-7.8(3 \mathrm{H}$, m, 6-, 7-, 8-H), $8.44(1 \mathrm{H}, \mathrm{dd}, J=8,2 \mathrm{~Hz}, 5-\mathrm{H}) .{ }^{13} \mathrm{C}-\mathrm{NMR}$ : Table III.

1-Methyl-2-[(4Z,7Z)-4,7-tridecadienyl]-4(1H)-quinolone (XIX)-Colorless oil $(9 \mathrm{mg}) . \mathrm{IR} v_{\max }^{\mathrm{KBr}} \mathrm{cm}^{-1}: 2980$, 2960, 2890, 1635, 1610, 1515, 1480, 1180, 765. UV $\lambda_{\max }^{\mathrm{MeOH}} \mathrm{nm}(\log \varepsilon): 213(4.41), 240$ (4.45), 321 (4.10), 334 (4.12). MS $\left.m / z: 337(\mathrm{M})^{+}(38), 186\left(\mathrm{M}-\mathrm{C}_{11} \mathrm{H}_{19}\right)^{+}(92), 173\left(\mathrm{M}-\mathrm{C}_{12} \mathrm{H}_{20}\right)^{+}(100), 144\left(\mathrm{M}-\mathrm{C}_{13} \mathrm{H}_{21} \mathrm{O}\right)^{+}(23) .{ }^{1} \mathrm{H}-\mathrm{NMR}(\mathrm{CDCl})_{3}\right)$ $\delta: 0.90\left(3 \mathrm{H}\right.$, br t, $\left.J=6 \mathrm{~Hz}, 13^{\prime}-\mathrm{H}_{3}\right), 1.2-1.4\left(6 \mathrm{H}\right.$, br m, 10' $\left.-11^{\prime}-, 12^{\prime}-\mathrm{H}_{2}\right), 1.80\left(2 \mathrm{H}, \mathrm{m}, 2^{\prime}-\mathrm{H}\right), 2.0-2.2\left(4 \mathrm{H}, \mathrm{m}, 3^{\prime}-, 9^{\prime}-\right.$ H), 2.7-2.8 (4H, m, 1'-, 6'- $\left.\mathrm{H}_{2}\right), 3.73\left(3 \mathrm{H}, \mathrm{s}, \mathrm{N}-\mathrm{CH}_{3}\right), 5.40\left(4 \mathrm{H}, \mathrm{m}, 4^{\prime}-, 5^{\prime}-, 7^{\prime}-, 8^{\prime}-\mathrm{H}\right), 6.24(1 \mathrm{H}, \mathrm{s}, 3-\mathrm{H}), 7.3-7.7(3 \mathrm{H}$, m, 6-, 7-, 8-H), 8.44 (1H, dd, $J=8,2 \mathrm{~Hz}, 5-\mathrm{H}) .{ }^{13} \mathrm{C}-\mathrm{NMR}$ : Table III.

Catalytic Hydrogenation of Quinolone Alkaloids_-A mixture of a solution of each compound (about $1 \mathrm{mg}$ ) in $\mathrm{EtOH}(1 \mathrm{ml})$ and $10 \% \mathrm{Pd}-\mathrm{C}(10 \mathrm{mg})$ as a catalyst was stirred for $12 \mathrm{~h}$ under hydrogen and then the catalyst was filtered off. The solution was concentrated and the product was identified as the side chain saturated compound by

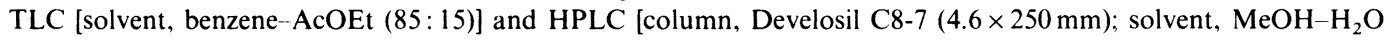
(90: 10); wavelength, $321 \mathrm{~nm}$ ] comparisons.

Lemieux-Johnson Oxidation of Quinolone Alkaloids-Osmium tetroxide ( $1 \mathrm{mg})$ and sodium periodate $(10 \mathrm{mg})$ in $\mathrm{H}_{2} \mathrm{O}(1 \mathrm{ml})$ were added to a solution of each compound (about $1 \mathrm{mg}$ ) in $\mathrm{H}_{2} \mathrm{O}(1 \mathrm{ml})$. The mixture was stirred for $24 \mathrm{~h}$ and extracted with ether. The ether layer, after being dried over anhydrous sodium sulfate, was treated with a saturated ether solution of 2,4-dinitrophenylhydrazine in large excess, and with concentrated $\mathrm{HCl}$. After washing of the reaction mixture with water twice, the ether was evaporated off, and the residue was dissolved in $\mathrm{MeOH}$ and shown to be identical with standard hydrazone, produced from an authentic sample of the aldehydes, by HPLC [column, YMC-Pack R-ODS-7 $(4.6 \times 250 \mathrm{~mm})$; solvent, $\mathrm{MeOH}-\mathrm{H}_{2} \mathrm{O}(90: 10)$; wavelength, $360 \mathrm{~nm}$ ] comparison.

Acknowledgement We thank Dr. S. Kadota, Research Institute for Wakan-Yaku (Oriental Medicines), Toyama Medical and Pharmaceutical University, for measurement of the $400 \mathrm{MHz}$ NMR spectrum, Dr. M. Uchida for measurement of mass spectra and Mrs. H. Kitamura for elemental analyses.

\section{References}

1) T. Reichstein, Helv. Chim. Acta, 15, 1100 (1932).

2) S. Arnott, A. W. Davie, and J. M. Robertson, J. Chem. Soc., 1961, 4183.

3) D. L. Dreyer, J. Org. Chem., 32, 3442 (1967).

4) Y. Hirose, Chem. Pharm. Bull., 11, 535 (1963).

5) T. Kubota, T. Kamikawa, T. Tokoroyama, and T. Matsuura, Tetrahedron Lett., 1961, 325.

6) J. Ahmad, K. Wizarat, K. M. Shamsuddin, and J. D. Connolly, Phytochemistry, 23, 1269 (1984).

7) R. D. Bennett and S. Hasegawa, Tetrahedron, 37, 17 (1981).

8) M. Nakatani, H. Takao, T. Iwashita, and H. Naoki, Bull. Chem. Soc. Jpn., 60, 2503 (1987).

9) T. Sugimoto, A. Ueno, S. kadota, C. Cui, and T. Kikuchi, Chem. Pharm. Bull., 36, 1237 (1988).

10) R. Tschesche and W. Werner, Tetrahedron, 23, 1873 (1967).

11) T. Kimimado, C.-F. Chang, S. Murakoshi, A. Sakurai, and S. Tamura, Agric. Biol. Chem., 40, 605 (1976). 